\section{Un caso de maloclusión Clase II división I de Angle tratado mediante mecánica de arco de canto (edgewise)}

A case report of class II division I malocclusion of Angle treated with cant arc mechanics (Edgewise)

\section{Leoncio Vladimir Menéndez Méndez}

Departamento Académico Estomatología Pediátrica. Facultad Odontología UNMSM. Lima - Perú

Correspondencia:

Mg. E. Leoncio Vladimir Menéndez Méndez

Facultad Odontología. UNMSM.

Av. Germán Amézaga s/n, Lima, 1 Perú. e-mail: leoncio.menendez@gmail.com

Palabras clave: Tratamiento de maloclusiones, clasificación de Angle, clase II. Ortodoncia.

\begin{abstract}
The present I articulate is developed the procedure of I diagnose and one treatment maloclusion class II division I of Angle, that development in the clinic of post degree of ortodoncia of the faculty of odontolgy of the UNMSM, by means of the technique edgewise, the same one that was created by the Dr. Edward Angle. The clinical case is described by means of the intraoral photographies extra and, the cefalometricos analyses and the obtained results can be evaluated by means of the cefalometricas superpositions, which indicates so much the craneofacial growth as the effects of the treatment.
\end{abstract}

Key words: malocclusion treatment. Angle classification. Class II. Ortodontics.

\section{Introducción}

El mecanismo de arco de canto es el resultado de varios intentos previos (arco básico E, arco de cinta, arco de perno y tubo) que experimentó y al final encontró la solución mecánica. Ya que el bracket que era de una ranura o slot de $0,022^{\prime \prime} \times 0,028^{\prime \prime}$ se inserta un alambre rectangular en la posición más estrecha o de canto, de ahí su nombre del aparato arco de canto. Un rasgo único del alambre rectangular en la ranura era que podían impartirse fuerzas de torsión o de torque en el arco de alambre para controlar la inclinación axial de los dientes. Con este aparato era posible mover los dientes en los tres planos del espacio con un simple arco de alambre. Para que el alambre rectangular pudiera mover los dientes a la línea de la oclusión, debía conformarse a la forma normal de la arcada y darle la torsión $\mathrm{y}$ torque adecuados para cada diente en particular.

Para realizar los movimientos de las piezas dentarias se estableció tres tipos de dobleces: los de primer, segundo y de tercer orden respectivamente.

Angle recomendó también el uso de alambres redondos de menores diámetros en los primeros estadios de tratamiento.

El aparato arco de canto distribuye las fuerzas a los dientes, por una variedad de medios. Los brackets y tubos reciben la mayor parte de las fuerzas. La selección y ubicación de estos vínculos influirá definitivamente en la dirección, distribución y cantidad de fuerza aplicada.

Los objetivos deberán ser claramente establecidos y sus metas de tratamiento serán eficientes y racionalmente ordenadas para permitir alcanzar el éxito en el resultado del tratamiento.

La mecánica edgewise es descrita como la técnica multibandas con brackets y auxiliares aplicados individualmente a cada diente.

En la práctica se usa arcos de alambres rectangulares de menor calibre, los cuales son usados para reducir la fricción del alambre dentro del bracket.

Para los procedimientos de tratamiento son necesarios consideraciones sobre el anclaje, para la eficiencia de cada aparato depende de la habilidad a establecer áreas estables de resistencia. Estos mismos alambres proveen una gran posibilidad de anclaje estacionario. El aparato de edgewise, adicionalmente, utiliza unidades de dientes con propósitos de anclaje.

Otro procedimiento para incrementar la eficiencia del arco edgewise envuelve el uso de loops verticales para abrir o cerrar los espacios, dependiendo de la corrección establecida.

Resortes abiertos o cerrados son usados para movimientos individuales de los dientes. Estos pueden colocarse alrededor del arco de alambre, o ellos pueden ser ligados en los tubos molares hacia los caninos o premolares. Aditamentos como los stops se sueldan mesial a los tubos molares. Ganchos para elásticos intermaxilares 
son soldados en el arco de alambre, o loops verticales para servir al mismo propósito puede se adaptado directamente en el arco de alambre.

Requerimientos técnicos no son simples para la mecánica edgewise. Debe de existir precisión en el aparato, y ciertos principios fundamentales no pueden ser ignorados.

Los brackets son alineados por cada arco de alambre. Si los brackets han sido posicionados apropiadamente entonces los dientes también estarán alineados adecuadamente.

Si los brackets son colocados más hacia incisal en los incisivos y más hacia cervical en los premolares. Esto tiende a deprimir los caninos y elevar a los premolares, una forma deseable de tratamiento particularmente efectivo en la corrección de una excesiva curva de Spee.

Han publicado diversos autores sobre tratamiento de maloclusiones de clase II division $1^{(1,2)}$, realizo estudio sobre la forma de arcos en sujetos con maloclusión clase II división sobre forma de arcos y estudio los efectos cefalometricos de dos modalidades de tratamiento para adolescentes con maloclusión clase II y overjets mayores a $4 \mathrm{~mm}$.

MUÑOZ 2005(3) Describe un caso clínico de maloclusión clase II con overbite profundo y asimetría dental en paciente adulto,, con un ANB de $7^{\circ}$, ángulo de plano mandibular $20^{\mathrm{a}}$ y una relación interincisal de $151^{\mathrm{a}} \mathrm{y}$ desviación de la línea media de 5 mm hacia la derecha

Otros autores estudiarons la duracion de tratamiento ${ }^{(4,5)}$, asi como estudios longitudinal ${ }^{(6)}$, JANSON $2004^{(7)}$ estudio sobre el éxito de tratamiento en pacientes con maloclucion clase II con extracciones de 2 premolares y 4 premolares.

BRIN $2003^{(8)}$ Este autor registro 138 casos de niños con maloclusión clase II con un overjet mayor de $7 \mathrm{~mm}$, los niños fueron tratados en la fase I con aparatología fija, en fase II con AEO $\mathrm{y}$ con bionator.

KING $2003^{(9)}$ Llegando a la conclusión que la hipótesis planteada no se verifica como que existen diferencias dentoalveolares que se obtuvieron entre la fase II y fase I de tratamiento de la maloclusión clase II.

Existen otros trabajos, que realizaron estudio comprativo sobre tratamiento ortodoncico con camuflage MIHOLIK $2003^{(10)}$ y Proffit

HICKS 2002(11), describe que la principal pregunta de la naturaleza de la corrección ortodoncica depende de la edad del paciente para empezar el tratamiento. Para analizar esta proposición fue la investigación de tres técnicas comunes de tratamiento de ortodoncia, Tweed edgewise, Begg con alambres ligeros y el arco recto, identificando sistemáticas diferencias en la naturaleza de la corrección en adolescentes.

KLOCKE 2002 ${ }^{(12)}$ juntamente con Ram NANDA, estudiaron los patrones esqueléticos de la clase II en la dentición decidua. El propósito el análisis cefalometrico longitudinal para identificar las características favorables y desfavorables del crecimiento.

BYRON 2002(13) estudiaron el tratamiento de la clase II con mordida profunda, con favorable crecimiento y excelente cooperación del paciente. El caso fue tratado con AEO y uso de elásticos intermaxilares, los resultados fueron una oclusión ideal y sin extracciones.

REDDY $2000^{(14)}$ estudio los cambios esquelétales y dentales en pacientes con maloclusión clase II división 1 mediante la terapia de Begg sin extracciones

FERRO 2000(15) realizo un estudio cefalometrito para evaluar los cambios antero posteriores y verticales producidos por la terapia Cetlin (lip bumper inferior, ACCO, AEO cervical) para corregir la maloclusión clase II división 1 durante la dentición mixta tardía.

Como se observa en los antecedentes anteriores descritos hay información en la literatura sobre tratamiento de maloclusión clase II

\section{Caso clínico}

Paciente de 12 años 7 mes de edad. Sexo: Masculino. Raza: Mestiza. E.G.S. BEG. Motivo de la consulta: "arreglarse los caninos"

Lado derecho lado izquierdo oclusal superior oclusal inferior. Se observa paciente mesofacial, con una biprotrusion labial, y contracción de los musculos del menton al cerrar los labios, paciente. En crecimiento intraoralmente presenta una maloclusion de clase II division I de Angle. (Fig. 1-4) (Fig. 5-8)
Análisis Radiográfico: Panorámico: con características normales

Análisis Cefalométrico: Notoriamente se observa que tienen buen potencial de crecimiento puberal.

Radiografía cefalometrica Panorámica cefalograma (Fig. 27,28)

Sumario de Diagnóstico:

Paciente masculino en dentición permanente, que viene a la consulta con extracciones de los primeros premolares superiores ya realizadas. Buen potencial de crecimiento. Presenta maloclusión de Clase II de Angle con relación esquelética de Clase I. Tipo de crecimiento mandibular a predominio horizontal. Se puede observar presencia de tejido adenoideo en la nasofaringe

Posición e inclinación de los incisivos: Protruidos e inclinados a vestibular.

Overbite $80 \%$; Curva de Spee pronunciada.; Discrepancia dentaria: discrepancia inferior severa $-9 \mathrm{~mm}$; Discrepancia de Bolton: Exceso en el sector antero inferior de $45 \mathrm{~mm}$; Perfil de tejidos blandos: Convexo.

Perfil óseo cóncavo; Línea media desviada 2 mm hacia lado derecha inferior; Tercio inferior aumentado (Fig. 18-22)

\section{Objetivos del Tratamiento}

Extracciones de las primeras premolares inferiores; Mantener la relación esquelética y corregir la maloclusión; Mantener la protrusión e inclinación de los incisivos; Corregir la relación canina y relación molar; Corregir la línea media inferio; Corregir la sobre mordida; Mejorar el perfil; Eliminar la discrepancia arco-diente inferio; Mejorar las asimetrías; Eliminar la discrepancia de Bolton.

Conseguir buen alineamiento e intercuspidación de primeras premolares

Resultados del Tratamiento:

Se corrigió la protrusion dentoalveolar superior. Se mejoró el perfil blando

Se mejoro la contracción de los músculos del mentón. Se corrigió la discrepancia arco diente. Se corrigió el over jet y el over bite. Se mantuvo la Clase I esquelética. Se corrigió la relación molar y canina a Clase I. Se mejoró la inclinación del incisivo superior y se aumentó levemente la protrusión e inclinación del incisivo inferior. Se mantuvo el perfil total convexo y se mejoró el perfil del tercio inferior de moderadamente convexo a ligeramente 
Fotografias extraorales del caso clínico inicial

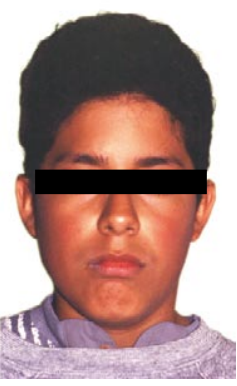

Fig. 1 Frontal Inicial

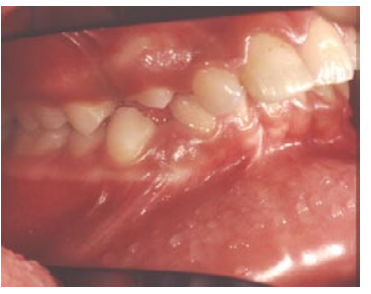

Fig. 5 Lado derecho

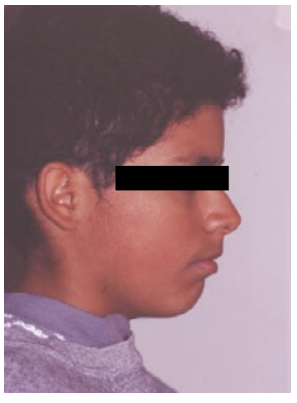

Fig. 2 Perfil Inicial

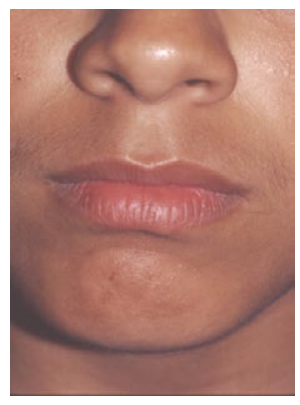

Fig. 3 Tercio inferior frontal

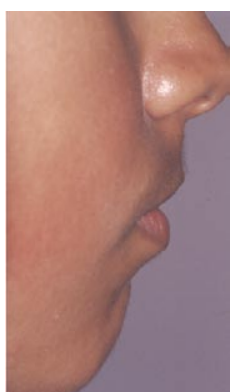

Fig. 4 Perfil

Fotografías intraorales del caso clínico inicial

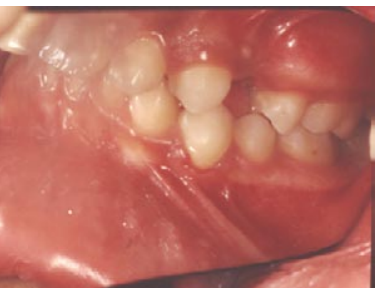

Fig. 6 Lado izquierdo

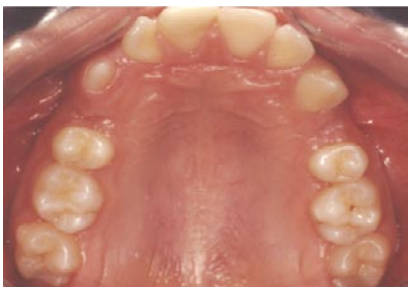

Fig. 7 Oclusal superior

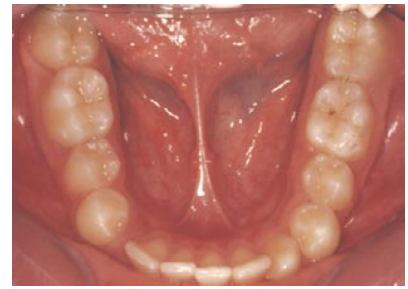

Fig. 8 Oclusal inferior

Fotografías extraorales del caso clínico final

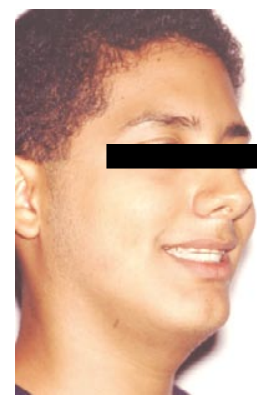

Fig. 9 Perfil 45 grados

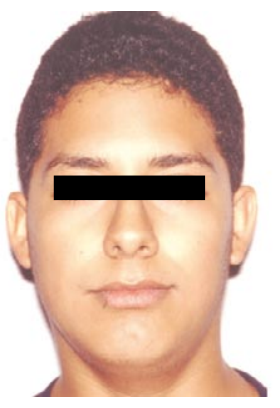

Fig. 10 Frontal

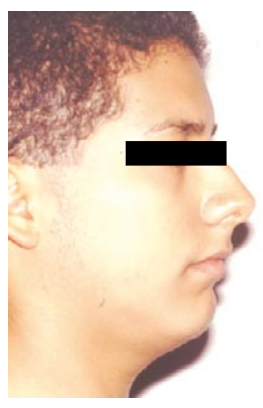

Fig. 11 Perfil total

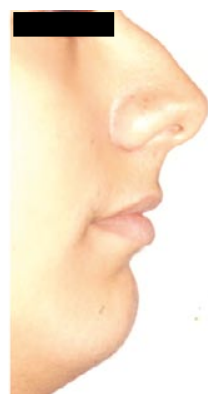

Fig. 12 Perfil tercio inferior

Fotografías intraorales del caso clínico final

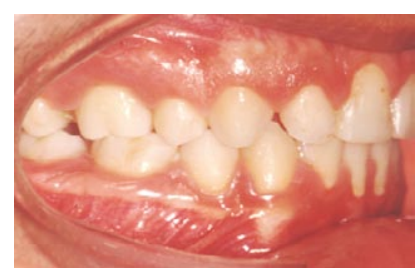

Fig. 13 Lado derecho

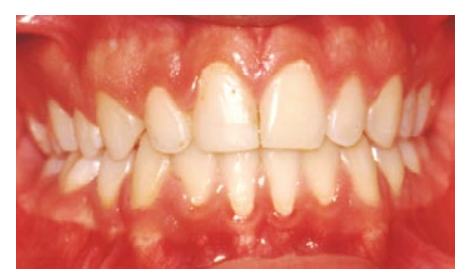

Fig. 14 Frontal

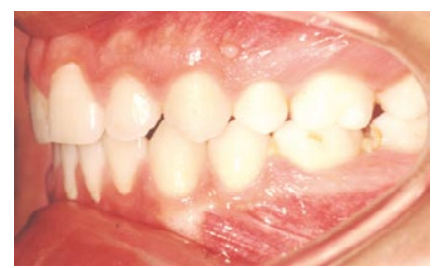

Fig. 15 Lado izquierdo

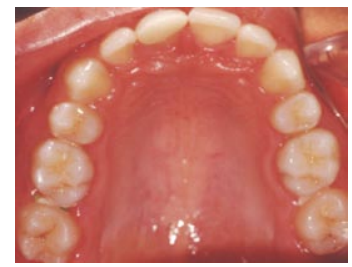

Fig. 16 Oclusal superior

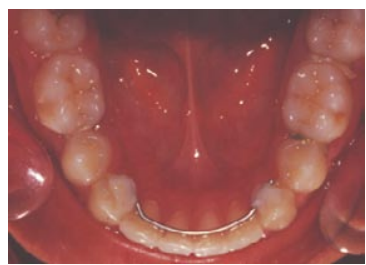

Fig. 17 Oclusal inferior 
Modelos de estudio del caso clínico inicial

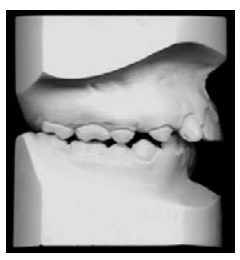

Fig. 18 Lado derecho

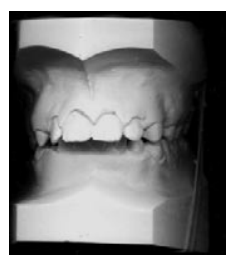

Fig. 19 Frontal

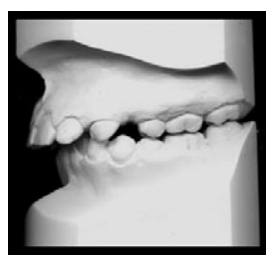

Fig. 20 Lado izquierdo

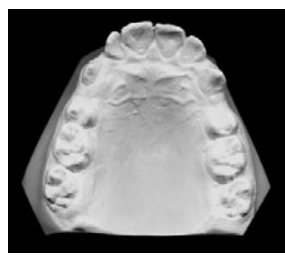

Fig. 21 Oclusal superior

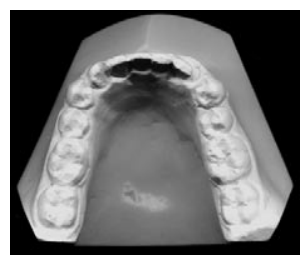

Fig. 22 Oclusal inferior

Modelos de estudio del caso clínico final

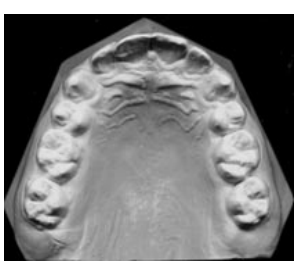

Fig. 23 Lado derecho

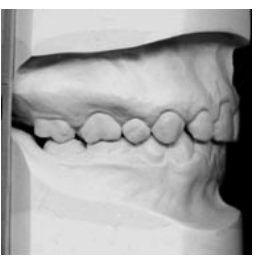

Fig. 25 Oclusal superior

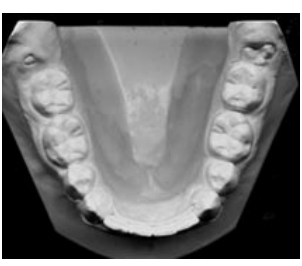

Fig. 24 Lado izquierdo

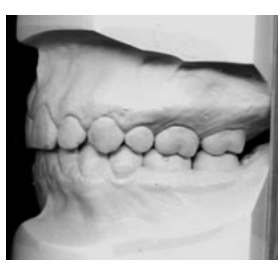

Fig. 26 Oclusal inferior
Radiografía y trazado inical

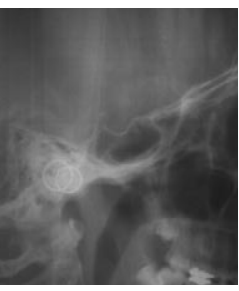

Fig. 27 Radiografia cefalométrica

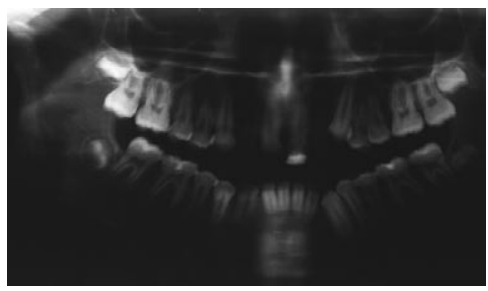

Fig. 28 Panorámica

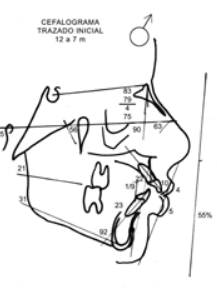

Fig. 29 cefalograma

Radiografía cefalométrica y trazado final

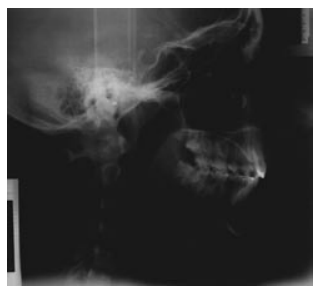

Fig. 30 cefalométrica

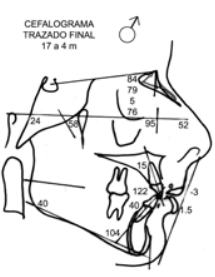

Fig. 31 Cefalograma convexo. (Fig. 9-12) (Fig. 13-17) El perfil óseo se mantuvo. Se corrigió la línea media inferior. Se mejoró las asimetrías molares. Se mejoró el tercio inferior de la cara. Se mejoraron la forma de los arcos superior e inferior. (Fig. 23-26)

Cefalométrica inicial y final. Cefalograma final (Fig. 30-31)

Evaluación mediante las superposiciones cefalométricas

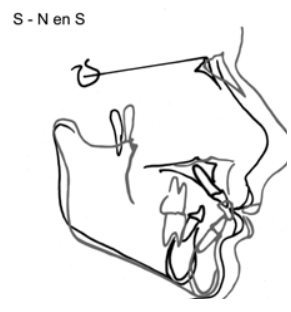

Fig.32

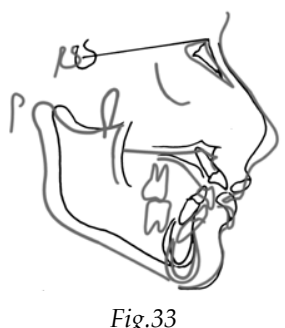

SuperposiciónS-N, Perfil facial S-N Registrado en Registrado en Sella. Cx. En sentido Nasion. Se observa Cx vertical y horizontal

Vertical y horizontal Nariz creció hacia delante y el mentón abajo y adelante

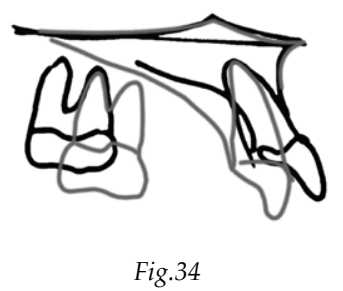

Superposición sobre el plano palatino con punto fijo en ENA. movimiento dentario en el maxilar superior se ha producido una mejor inclinación del incisivo superior hacia palatino. La molar muestra una leve extrusión y mesializacion moderada.

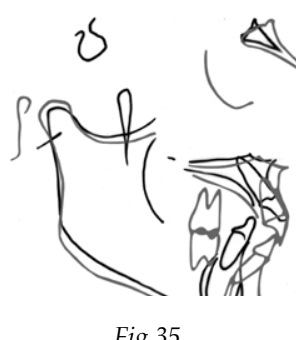

Crecimiento antero posterior de la maxila registrado en PTM la maxila muestra un crecimiento horizontal significativo

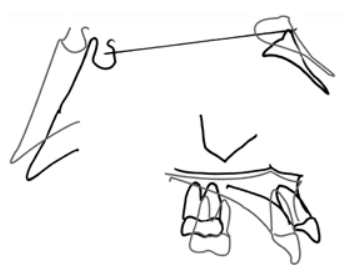

Fig.36

Crecimiento Vertical de la Maxila Registrado en el Key Ridge Ha existido un ligero, crecimiento vertical. Movimiento Dentario Superior. El incisivo superior ha realizado un movimiento en sentido palatino. Así como la molar ha seguido migrando hacia mesial

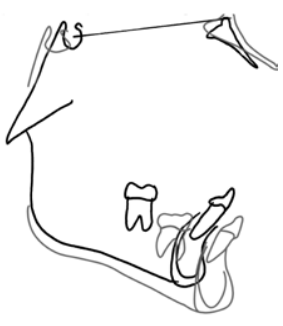

Fig.37 
Crecimiento Mandibular Registrado en Articular. Se observa un crecimiento mandibular mayor en sentido horizontal que en sentido vertical. se observa que después del tratamiento la mandíbula opto por un crecimiento mas horizontal que vertical. Ha habido una remodelación ósea del mentón.

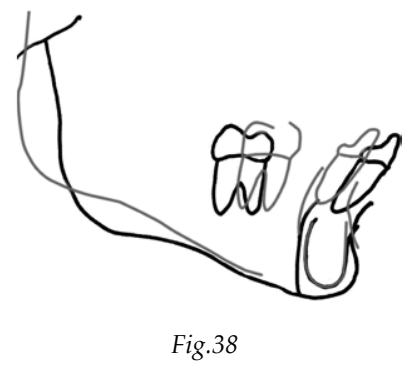

Superposición en la sínfisis mentoniana Movimiento Dentario Inferior .El incisivo inferior se ha ubicado más hacia lingual que al inicio y hubo migración mesial de la molar inferior.

\section{Discusión}

En los trabajos que se realizaron en población escolar de 11 a 16 anos de obtuvo un porcentaje de $12 \%$ de maloclusion clase II ${ }^{(16)}$, y autores como Proffit, determinaron que los tratamientos podrían ser conservando la s piezas dentarias (sin extracciones) o en casos que el problema se vea afectando la estética, el tratamiento seria con extracciones Esto debido al fuerte influencia del factor hereditario en la etiología de las maloclusiones ${ }^{(17,18,19,20,21,22,23,24)}$.

El presente caso se desarrollo con extracciones las primeras 4 premolares, con el uso de aparatología extraoral, para acción de anclaje así como para corregir la relación molar clase I. Se instalaron la aparatología de brackets estándar edgewise y alambres de acero inoxidable empezando con multiloops 0,016 " hasta los $0,020^{\prime \prime}$ para luego del nivelamiento y alineamiento respectivo cambiar a alambres $0,018^{\prime \prime} \times 0,022^{\prime \prime}$ para llegar a la coordinación de arcos con alambres de finalización 0,019" x $0,025^{\prime \prime}$. Se lograron los objetivos de tratamiento establecidos, y el tiempo de tratamiento duro aproximadamente 24 meses.

\section{Conclusiones}

a Los incisivos inferiores se intruyeron.

b Los incisivos se palatinizaron.

c Las raíces de las piezas dentarias están paralelas en las áreas adyacentes a la extracción. d No ha habido reabsorción radicular

e Se aplicó torques en todas las piezas.

\section{Referencias bibliográfica}

1. NIE, Q Lin J. “ A comparison of dental arch forms between class II division 1 an normal occlusion by Euclidean distance matrix analysis" AJODO April 2006; Vol. 124(4): 528-535

2. DARCY C. "A cephalometrics comparison of two treatment modalities for adolescents patient with class II malocclusion: cervical headgear versus maxillary first premolar extraction" AJODO Marc 2006; Vol. 129(3): 448

3. KAGEYAMA,Toruetal.“Amorphological study of the relationship between arch dimension and craniofacial structures in adolescent with class II division 1 malocclusion and various facial types" AJODO March 2006; Vol. 124(3): 368375.

4. POPOVICH, Kurt et al "Predictors for class II treatment duration" AJODO Marc 2005; Vol. 127(3): 293-300

5. HERO et al. "Duration of treatment and mandible lengthening by means of distracction or bilateral sagittal split osteogenics pacients wiht Angle malocclusion class II." AJODO 2005; Vol. 127(11): 25-29

6. MARCIA, Sandra et al. "Influence of extraction and nonextraction orthodontic treatment in japonessebrasilian with class I and class II division 1 malocclusion" AJODO 2005; Vol. 127(1): 30-36

7. JANSON,“"Class II treatment success rate in 2 and 4 premolar extraction protocols" AJODO 2004; Vol. 12(4): 472-479.

8. BRIN Llam et al "External apical root resortion in class II malocclusion A retrospective review of 1 versus 2 phases treatment" AJODO; 2003: 151156.

9. KING, Gregory et al. "Comparison of peer assessment ratings (PAR) from 1 phase and 2 phase treatment protocols for class II malocclusion" AJODO May 2003; Vol. 123(5)

10. NITHOLIK, Colin A. and PROFFIT William et al. "Long-term followup of class II adults treatment with orthodontic camouflage: A comparison with orthognatic surgery outcomes" AJODO march 2003; Vol. 123(3): 266278

11. HICKS , Joseph "Influence of age, sex and orthodontic treatment modality on the correction of class II malocclusion" AJODO; Dec 2002: 676

12.KLOCKE, A, NANDA, Ram, NIEKE,Kahl" Skeletal class II patterns in primary dentition" AJODO June 2002; Vol. 121(6): 516-601.

13. BYRIN, Albia Hammond “Treatment o class II malocclusion with deep overbite" AJODO may 2002; Vol. 121(5): 531-537

14. REDDY, Pramita, et al "Skeletal and dental changes with no extraction Begg mechanic therapy in patients with class II division 1 malocclusion" AJODO Dec 2000; Vol. 118(6): 641-648

15. FERRO, Fabrizia , et al "Saggittal and vertical change after treatment of class II division 1 malocclusion according to the Cetlin method" AJODO 2000; Vol. 118(2): 150-158

16. Menéndez Leoncio," Estudio comparativo de tres índices de maloclusiones OMS $<$ AIO $<$ AGUILA ; en un grupo de escolares de 11 a 16 anos de edad de sexo femenino en la ciudad de Lima" Tesis maestría en Estomatología , Lima UNMSM 1999

17. Profflt W.: Ortodoncia. Teoría y Práctica. 2da Edición. Mosby/Doyma Libros. 1994

18. Ricketts R, Fairchield R and Steele C: Forum on the tonsil and adenoid problem in orthodontics. 1968; (54): 485-513

19. Harvold E, Vangervik K: Primate experiments on oral sensatíon and dental malocclusion. Am ]ourn of Orthod 1973; (63): 494-508

20. Linder-Aronson 5: Effects of adenoídectomy on dentitíon ami nasopharynx. Am Journ of Orthod 1974; (85): 1-15

21. Salinas C: Genética craneofacial. Public Científica 378. Organización Panamericana de la salud. 1979

22. Marcotte, "Biomecánica" , ediciones científicas y Técnicas S.A., Masson Salvat, 1992.

23. Graber Vanarsdall "Ortodoncia" Principios Generales y Técnicas. Buenos Aires: Editorial Médica Panaméricana. 2003

24. Van der Linden "Ortodoncia con aparatólogia fija" Venezuela: Actualidades Medico Odontológicas Latinoaméricana. 1999

Recibido :04-06-2008

Aceptado para publicación: 05-08-2008 